\title{
Aspectos biológicos de Archaeoprepona demophon muson (Fruhstorfer, 1905) (Lepidoptera: Nymphalidae, Charaxinae) en la Amazonía peruana
}

\section{Biological aspects of Archaeoprepona demophon muson (Fruhstorfer, 1905) (Lepidoptera: Nymphalidae, Charaxinae) in the Peruvian Amazon}

\section{Joel Vásquez Bardales ${ }^{1 *}$, Ricardo Zárate Gómez ${ }^{1}$, Andrés Fernández Sandoval ${ }^{2}$, Pedro Vela García ${ }^{1}$, Julio Pinedo Jiménez ${ }^{3}$, Juan José Ramírez Hernández ${ }^{4}$, Gerardo Lamas ${ }^{4}$}

1 Instituto de Investigaciones de la Amazonía Peruana (IIAP), Programa de Investigación en Biodiversidad Amazónica (PIBA), Apartado 784, Iquitos, Perú 2 Instituto Nacional de Innovación Agraria -INIA -, Estación Experimental Agraria "San Roque". Calle San Roque N²09, San Juan Bautista, Loreto, Perú. 3 Universidad Nacional de la Amazonía Peruana, Facultad de Agronomía. Calle Pevas S/N, Iquitos, Perú.

4 Museo de Historia Natural, Universidad Nacional Mayor de San Marcos, Av. Arenales 1256, Lima-11, Perú.

* Autor para correspondencia

Email Joel Vásquez Bardales: jvasquez@iiap.org.pe

Email Ricardo Zárate Gómez: rzarate@iiap.org.pe

Email Andrés Fernández Sandoval: afernandez@inia.gob.pe

Email Pedro Vela García: pvelacaligo@gmail.com

Email Julio Pinedo Jiménez: jupiji@hotmail.com

Email Juan José Ramírez Hernández: macrodontia@hotmail.com

Email Gerardo Lamas: glamasm@unmsm.edu.pe

\begin{abstract}
Resumen
El objetivo de este trabajo fue elucidar el ciclo biológico de la mariposa diurna Archaeoprepona demophon muson, determinar las especies de plantas con las que se relaciona, caracterizar su hábitat y contribuir al conocimiento sobre sus enemigos naturales. El trabajo de campo se desarrolló en dos áreas cercanas a la ciudad de lquitos (Perú). Para describir el ciclo biológico se utilizó 20 huevos recientemente depositados en las hojas de su planta hospedera. La duración del ciclo, desde huevo hasta adulto fue de $85.40 \pm 4.66$ días en condiciones de laboratorio. El periodo de huevo se extendió por $5.60 \pm 0.52$ días. La larva pasa por cinco estadíos: el primero duró $8.61 \pm 0.77$ días, el segundo $6.12 \pm 0.68$ días, el tercero $11.44 \pm 0.73$ días, el cuarto $8.13 \pm 0.34$ días, y el quinto $27.37 \pm 1.29$ días. El periodo de la prepupa duró $3.60 \pm 0.51$ días y el de pupa $14.13 \pm 2.62$ días; los adultos nacieron aproximadamente entre las 9:00 a 11:00 h. La hembra vivió 41.00 \pm 6.35 días y el macho $19.25 \pm 4.49$ días. Las plantas alimenticias utilizadas fueron Siparuna bifida (Poepp. \& Endl.) A.DC., Pouteria caimito (Ruiz \& Pav.) Radlk. y Musa x paradisiaca L. Registramos dos enemigos naturales, una araña y un hongo.
\end{abstract}

Palabras clave: Amazonía de Perú; Ciclo biológico; Manejo de mariposas; Enemigo natural; Plantas hospederas.

\section{Citación:}

Vásquez Bardales J., R. Zárate Gómez, A. Fernández Sandoval, P. Vela García, J. Pinedo Jiménez, J.J. Ramírez Hernández, G. Lamas. 2017. Aspectos biológicos de Archaeoprepona demophon muson (Fruhstorfer 1905) (Lepidoptera: Nymphalidae, Charaxinae) en la Amazonía peruana. Revista peruana de biología 24(3): 249 - 254 (octubre 2017). doi: http:// dx.doi.org/10.15381/rpb.v24i3.13906

$\begin{array}{ll}\text { Presentado: } & 25 / 08 / 2016 \\ \text { Aceptado: } & 14 / 07 / 2017\end{array}$

Publicado online: $28 / 10 /$
Información sobre los autores:

JVB y RZG diseñaron y redactaron el manuscrito. JVB y JPJ realizaron la evaluación del ciclo de vida. RZG colectó e identificó muestras botánicas. AFS y PVG realizaron la colecta y las observaciones del comportamiento de la mariposa. JJRH y GL, realizaron la identificación taxonómica de la mariposa y la revisión general del manuscrito. Todos los autores revisaron y aprobaron el manuscrito.

Los autores no incurren en conflictos de intereses.

Fuentes de financiamiento: Innóvate-Perú proyecto "Modelos tecnológicos de crianza de 10 especies de mariposas diurnas para su aprovechamiento en bionegocios en la Región Loreto" 


\begin{abstract}
The aim of this study was to elucidate the life cycle of the butterfly Archaeoprepona demophon muson, determine the species of plants to which it relates, characterize its habitat and contribute to the knowledge about their natural enemies. Fieldwork was conducted in two areas near lquitos (Peru). For the description of the life cycle 20 eggs laid on leaves of it host plant were used. The cycle from egg to adult lasted $85.40 \pm 4.66$ days under laboratory conditions. The egg period took $5.60 \pm 0.52$ days. The larva goes through five stages: the first one lasts $8.61 \pm 0.77$ days; the second $6.12 \pm 0.68$; the third $11.44 \pm 0.73$; the fourth $8.13 \pm 0.34$; and the fifth $27.37 \pm 1.29$. The prepupa lasted $3.60 \pm 0.51$ days and the pupa $14.13 \pm 2.62$; adults were born approximately between 9:00 to $11: 00 \mathrm{~h}$. The female lived $41.00 \pm 6.35$ days and the male $19.25 \pm 4.49$ days. The host plants were Siparuna bifida (Poepp. \& Endl.) A.DC., Pouteria caimito (Ruiz \& Pav.) Radlk. and Musa × paradisiaca L. We report two natural enemies, a spider and a fungus.
\end{abstract}

Keywords: Biological cycle; Butterfly management; Host plants; Natural enemies; Peruvian Amazon.

\section{Introducción}

La especie Archaeoprepona demophon (Linnaeus, 1758) se distribuye en América Central y del Sur, desde México hasta Argentina (Thomas 1991; Lamas et al. 1997, Salazar 2011, Checa et al. 2009, Lazzeri et al. 2012). Su subespecie Archaeoprepona demophon muson (Fruhstorfer, 1905) ocurre desde el norte de Venezuela hasta Bolivia y el oeste de Brasil (Robbins et al. 1996, Campos \& Ramírez 2005, Henao 2006, Bonfantti et al. 2013, Calero-Mejía et al. 2014). Las larvas de A. d. muson son polífagas y se han reportado varias especies de plantas hospederas en diferentes países del Neotrópico Beccaloni et al. (2008). González (2006) registra cuatro géneros de hospederos: Aniba, Rollinia, Annona y Citrus en Tarapoto, Perú, y Vásquez et al. (2017) citan a Siparuna bifida en Iquitos, Perú. En cuanto a su biología, González (2006) estudió su preferencia alimenticia en Tarapoto, indicando la variación de su ciclo biológico de acuerdo a dos dietas vegetales. Esta mariposa posee un buen potencial para los bionegocios (confección de artesanías), siendo comercializada en países como Estados Unidos, Bélgica y Austria con precios que oscilan entre los 3 a 7 dólares por unidad y el par de calidad A1 (hembra y macho) a 19.20 dólares. Son poco conocidos los aspectos biológicos de esta mariposa, hecho que obstaculiza su crianza masiva y sostenible en cautiverio y semicautiverio. Este articulo pretende contribuir al conocimiento de los aspectos biológicos de $A$. demophon muson utilizando como alimento para sus larvas las hojas de Siparuna bifida (Siparunaceae) en condiciones de cautiverio para desarrollar crianzas masivas orientadas a su conservación, educación ambiental y bionegocios (turismo y artesanía).

\section{Material y método}

Área de estudio.- El trabajo fue realizado desde febrero 2014 hasta octubre 2015, en el Centro de Investigaciones Allpahuayo (CIA) y la comunidad de San Rafael. El Centro de Investigaciones Allpahuayo (CIA) está ubicada aproximadamente a 26 $\mathrm{km}$ de la ciudad de Iquitos, en el distrito de San Juan Bautista, provincia de Maynas, departamento de Loreto (3 $38^{\circ} 01.99^{\prime \prime}$; $\left.73^{\circ} 25^{\prime} 08.86^{\prime \prime} \mathrm{W}\right)$, su clima presenta temperatura media anual mayores a $24^{\circ} \mathrm{C}$ y precipitaciones promedio anuales mayores a $2400 \mathrm{~mm}$, distribuidas sin una estación seca definida; la estación más lluviosa se presenta de noviembre a mayo. la Comunidad

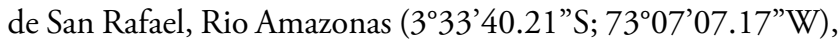
su clima de la zona es cálido y húmedo, con temperatura media de $26^{\circ} \mathrm{C}$ y precipitación promedio anual de $2900 \mathrm{~mm}$, siendo las épocas de mayor lluvia entre enero y febrero (IIAP, 2008). Cuenta con mariposarios experimentales, plantaciones de plantas hospederas y zonas de bosque primario donde se efectuaron las observaciones (Fig. 1).

Evaluación de las plantas hospederas y comportamiento. Para evaluar las plantas hospederas se utilizó un mariposario de $500 \mathrm{~m}^{2}$ con $4 \mathrm{~m}$ de altura cubierto con malla raschell de $65 \%$ de sombra y que contenía plantas de diferentes familias botánicas. Para obtener las hembras adultas, se utilizó cebos (rodajas de plátanos maduros fermentados en jugo de tallos de caña de azúcar), colocadas sobre estacas de $1.20 \mathrm{~m}$ de altura en diferentes tipos de hábitat, tales como bosque primario de la reserva comunal de San Rafael, bosque intervenido de terraza media y bosque intervenido de terraza baja. Luego de atrapar las hembras, se colocaron en el interior del mariposario, donde procedieron a ovipositar. Las larvas fueron recolectadas en el mariposario manualmente cuando se alimentaban de las hojas de su hospedero y fueron colocados en envases plásticos. Luego se transportaron al laboratorio para ser confinadas en envases apropiados y se las alimentó hasta el final de su desarrollo larval. Los adultos obtenidos fueron preparados en el laboratorio para su posterior identificación. Se efectuaron observaciones y se registraron datos de su comportamiento alimenticio en condiciones naturales y en cautiverio (mariposario), su comportamiento reproductivo, de vuelo, oviposición y número de huevos depositados.

Evaluación del ciclo biológico.- El ciclo biológico fue registrado en el laboratorio a una temperatura que osciló entre los 22 a $32^{\circ} \mathrm{C}$, a partir de veinte huevos recolectados inmediatamente después de la oviposición de la hembra dentro del mariposario. Las larvas emergidas se individualizaron en envases plásticos de $1 \mathrm{~L}$.

Se les proporcionó hojas de Siparuna bifida para su alimentación. El recambio del alimento fue diario debido a que la hoja se seca después de las 24 h. La eliminación de las excretas y el alimento sobrante fue diario, evitando así factores adversos de humedad y proliferación de entomopatógenos. A partir del quinto estadío se colocaron pedazos de rama dentro del envase ya que la larva abandona la hoja y reposa en la rama. Desde allí sale a comer; luego de completar su desarrollo larval empupa en la rama o cerca al ápice de la hoja.

Al día siguiente del empupamiento las crisálidas fueron retiradas de las ramas y se pegaron en la tapa del recipiente plástico para favorecer el estiramiento alar en los adultos emergentes. 
El número de estadíos fue determinado a través de las mudas larvales y la medición del ancho de las cápsulas cefálicas.

Luego de obtener los adultos, fueron liberados en un mariposario de $500 \mathrm{~m}^{2}$, que contenía plantas de Siparuna bifida. Para la alimentación de los adultos se acondicionó comederos con rodajas del fruto maduro de Musa x paradisiaca (plátano) macerado en jugo de Saccharum officinarum L. (caña de azúcar) y untadas con miel de abeja.

Enemigos naturales.- Para identificar los posibles enemigos naturales se recolectaron muestras de huevos, larvas y pupas del mariposario. Estas muestras fueron confinadas en envases herméticos de plástico en condiciones de laboratorio y diariamente se evaluó la presencia de sus enemigos naturales.

Identificación taxonómica.- La determinación taxonómica de las mariposas fue realizada utilizando claves especializadas y comparación directa con muestras depositadas en el Museo de Historia Natural, Universidad Nacional Mayor de San Marcos, Lima, Perú (MUSM). Las muestras entomológicas están depositadas en el laboratorio de crianza de mariposas del Instituto de Investigaciones de la Amazonía Peruana (IIAP).

La identificación de las plantas hospederas y alimenticias se realizó siguiendo el procedimiento usual de herborización (Judd et al. 1999), y con las claves taxonómicas de Amasifuén \& Zárate (2005), Vásquez \& Rojas (2004), Ribeiro et al. (1999), Vásquez (1997) y Gentry (1993). Las muestras están depositadas en el Herbario del IIAP.
Fotografías.- Todos los registros fotográficos fueron realizados por uno de nosotros (JV) con una cámara digital de 18.5 megapixeles y zoom óptico de $20 \mathrm{X}$.

\section{Resultados y discusión}

Evaluación de las plantas hospederas y comportamiento.En nuestros muestreos Archaeoprepona demophon muson solo fue encontrada en bosques intervenidos de terrazas medias, sin embargo puede habitar áreas de alta, media y baja perturbación (Campos \& Ramírez 2005, Calero-Mejía et al. 2014).

La mayor actividad se da entre las 08 y 11 h. Efectúan vuelos muy rápidos y reposan en las copas de los árboles, solo bajan para alimentarse de frutas caídas en estado de fermentación o aquellas que se encuentran en los arboles tales como Pouteria caimito. Los machos además son atraídos por minerales del suelo húmedo y excretas de animales. Similar comportamiento fue observado por Muyshondt (1976) quien reporta además que la subespecie $A$. demophon centralis (Fruhstorfer, 1905) también puede alimentarse de resinas segregadas por heridas de los árboles. En cautiverio se alimenta entre las 08 y $13 \mathrm{~h}$ y en las tardes de 14 a $15 \mathrm{~h}$ de fruta fermentada y de sales minerales del suelo.

La hembra deposita sus huevos en el haz y en el envés de las hojas pero con mayor frecuencia en el envés entre las 9 a 12 $\mathrm{h}$ y en las tardes de 14 a $15 \mathrm{~h}$. Muyshondt (1976) indica que las hembras de $A$. $d$. centralis depositan sus huevos solo en la superficie inferior (envés) de las hojas maduras de su hospedera.

El cortejo se inició a las 10:30 h. El macho persigue a la

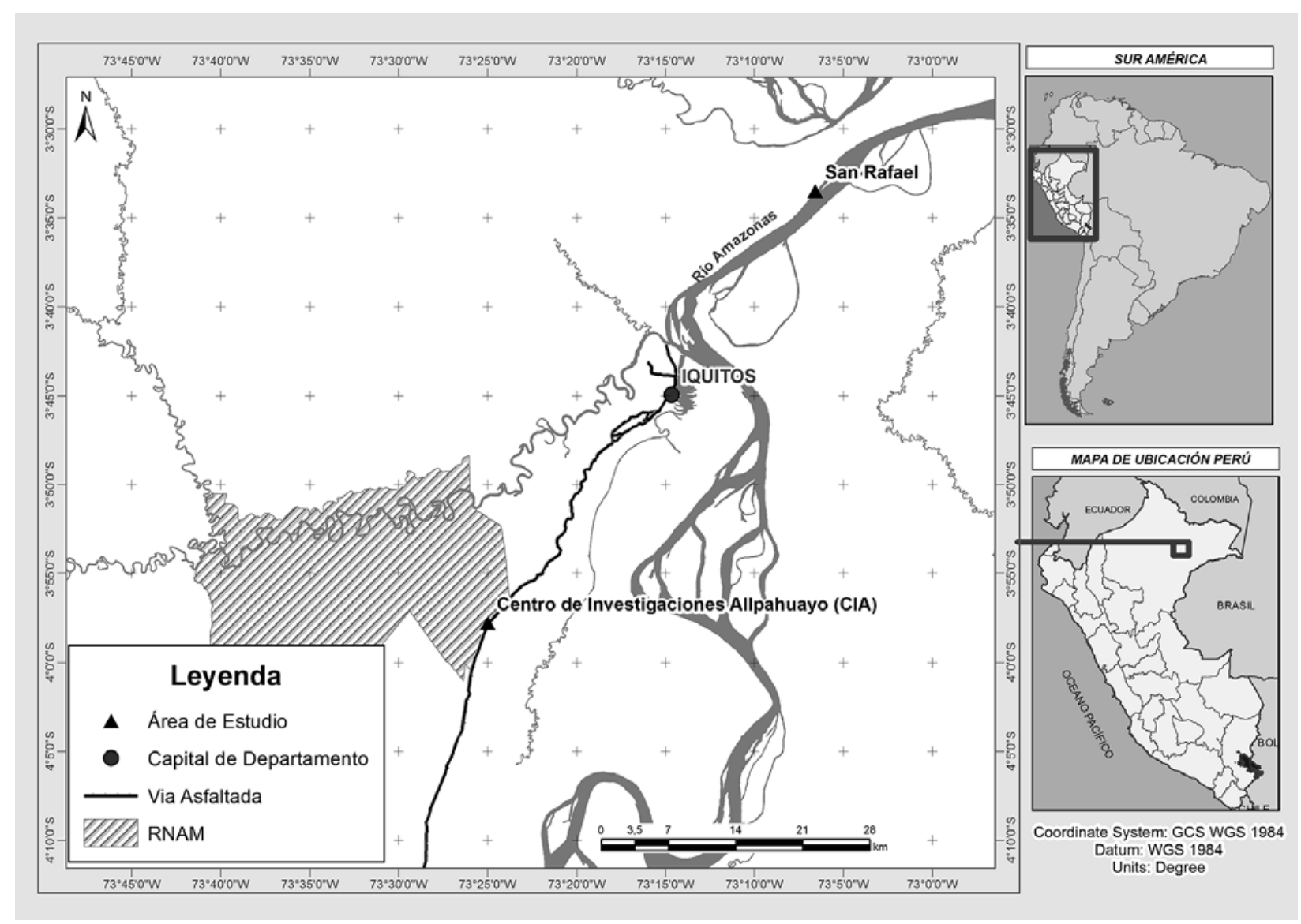

Figura 1. Ubicación del área de estudio: Comunidad de San Rafael y Centro de Investigaciones Allpahuayo, Loreto, Perú. 
hembra con vuelos muy rápidos y ambos se detienen en las copas de los árboles, el macho se acerca lentamente por detrás de la hembra, si ella no está receptiva continua la persecución y persiste hasta alcanzar su objetivo. Sólo se logró observar una cópula a las 11:56 h y ocurrió en la copa de un árbol a una altura aproximada de $4 \mathrm{~m}$. Cuando la hembra va a depositar sus huevos revolotea alrededor de la planta hospedera (Siparuna bifida) dando vuelos ligeros y deposita los huevos en $2.9 \pm 0.91 \mathrm{seg}(\mathrm{n}=20)$, repitiendo la oviposición durante el día y en días consecutivas en la hoja elegida, habiéndose encontrado hasta un máximo de 19 huevos ovipositados individualmente en una sola hoja.

\section{Ciclo biológico.-}

- Los huevos (Fig. 2a) son redondos con superficie lisa, de coloración rosada que se torna blanca paulatinamente, debido a la larva blanquecina que se desarrolla en el interior. En el tramo final, antes de la emergencia de la larva, se puede notar a través del corion las mandíbulas negras y pequeñas bandas oscuras con puntos negros en el dorso. Mide $2.0 \mathrm{~mm}$ y dura $5.60 \pm 0.52$ días. El color del huevo y el periodo de duración es muy similar a lo descrito por González (2006). Sin embargo, el periodo de duración es ligeramente menor al de $6.63 \pm 0.98$ días y diferente a los huevos de $A$. d. centralis que son blancos, tal como referido por Muyshondt (1976).

- La larva pasa por cinco estadíos. Las medidas de los estados de desarrollo y del ancho de la capsula cefálica para cada estadío se indican en la Tabla 1.

- En el primer estadío la larva (Fig. 2b) es translúcida, la cabeza presenta líneas marrón oscuro en forma de "M". En el dorso se observan tres estructuras circulares ubicadas una en el segmento T3 y dos en el A2. El primero es de posición central y los dos posteriores dorso-laterales, unidos por una línea oscura formando un triángulo. El resto del cuerpo es angosto y termina en una pequeña cola. Tiene una duración de $8.61 \pm 0.77$ días. Este periodo es menor a lo encontrado por González (2006) quien reporta una duración de $10.12 \pm 2.75$ días, en larvas alimentadas con hojas de Rollinia sp. y $9.20 \pm 2.75$ días en larvas alimentadas con hojas de Aniba sp. La larva del primer estadío después de emerger no consume la cáscara del huevo; se dirige hacia el ápice de la hoja, e inicia su alimentación inmediatamente consumiendo el borde del ápice dejando una parte de la vena donde se refugia. A medida que pasan los días, la larva va construyendo un escondite con restos de hojas secas que une con su seda; desde allí sale a alimentarse y luego vuelve nuevamente a su refugio.
- El segundo estadío (Fig. 2c) exhibe diversas tonalidades. La cabeza presenta puntos marrones, se mantiene la línea "M" y aparecen dos pequeños cuernos. El dorso es marrón claro. Las tres estructuras circulares ubicadas en los segmentos T3 y A2 crecen en forma de espolones y la zona se abulta en forma de joroba. La parte abdominal se mantiene angosta y la cola es notoria. Dura $6.12 \pm 0.68$ días. Este periodo es menor a lo encontrado por González (2006) quien reporta $9.64 \pm 3.27$ días para larvas alimentadas con hojas de Rollinia sp. y $7.82 \pm 1.62$ días con hojas de Aniba sp.

- En el tercer estadío la larva es marrón oscuro, la joroba es notoria. Las tres estructuras de la joroba (espolones) crecen. La primera estructura del segmento T3 se proyecta hacia adelante y las dos posteriores son pronunciadas, triangulares y curvadas hacia atrás. La cabeza presenta los cuernos más grandes y tiene una duración de 11.44 \pm 0.73 días, mayor a lo encontrado por González (2006) quien indica $9.79 \pm 2.60$ días para larvas alimentadas con hojas de Rollinia sp. y $8.37 \pm 1.77$ días con hojas de Aniba sp.

- En el cuarto estadio (Fig. 2d), La larva mantiene las características del estadío anterior y la joroba es prominente. Los cuernos de la cabeza crecen y se doblan hacia atrás. La duración es de $8.13 \pm 0.34$ días, mucho menor a lo encontrado por González (2006) quien reporta $17.44 \pm$ 4.88 días para larvas alimentadas con hojas de Rollinia sp. y $14.69 \pm 3.06$ días, alimentadas con hojas de Aniba sp.

- El quinto estadío (Fig. 2e) se caracteriza por el cambio en los espolones del segmento A2 que adquieren una forma circular a manera de ojos, con el borde interno violeta. La cabeza y la zona de la joroba son marrón oscuro y el dorso de la zona abdominal marrón claro con finas líneas oscuras. La parte final del abdomen y la cola son marrón oscuro. Los cuernos de la cabeza crecen y se mantienen doblados hacia atrás. La cola es muy notoria y larga. Al final de su desarrollo la larva alcanza una longitud de 80 $\mathrm{mm}$ y la joroba mide $15 \mathrm{~mm}$ de ancho. La duración fue $27.37 \pm 1.29$ días, menor a lo encontrado por González (2006), de $39.15 \pm 6.21$ días, con hojas de Rollinia sp. y $32.89 \pm 4.27$ días con hojas de Aniba sp. En este estadío la larva abandona la punta de la hoja y sube a posarse sobre una rama. Desde allí busca las hojas para alimentarse.

- Prepupa (Fig. 2f). En este estadío la larva se torna de color verde siendo la zona torácica (joroba) y el cuello verde intenso; a partir del segundo día esta coloración se intensifica y al final la larva se adhiere a un sustrato a

Tabla 1: Medidas de los estados, estadíos larvales y el ancho de la cápsula cefálica durante el estudio del ciclo biológico de Archaeoprepona demophon muson en laboratorio (febrero 2014 a octubre 2015)

\begin{tabular}{|c|c|c|c|c|c|c|}
\hline Estado & Estadío & Longitud (mm) & $\mathrm{SD}$ & $\begin{array}{c}\text { Cápsula } \\
\text { cefálica }(\mathrm{mm}) \\
\end{array}$ & SD & $\mathbf{n}$ \\
\hline \multirow{6}{*}{ Huevo } & & 2.00 & 0.00 & & & 20 \\
\hline & Larva I & 5.00 & 0.00 & 1.00 & 0.00 & 19 \\
\hline & Larva II & 10.05 & 0.28 & 2.00 & 0.00 & 18 \\
\hline & Larva III & 20.70 & 1.13 & 2.50 & 0.00 & 16 \\
\hline & Larva IV & 37.50 & 2.06 & 4.00 & 0.00 & 16 \\
\hline & Larva V & 50.60 & 1.10 & 5.50 & 0.00 & 15 \\
\hline Pupa & & 42.20 & 2.62 & & & 15 \\
\hline
\end{tabular}



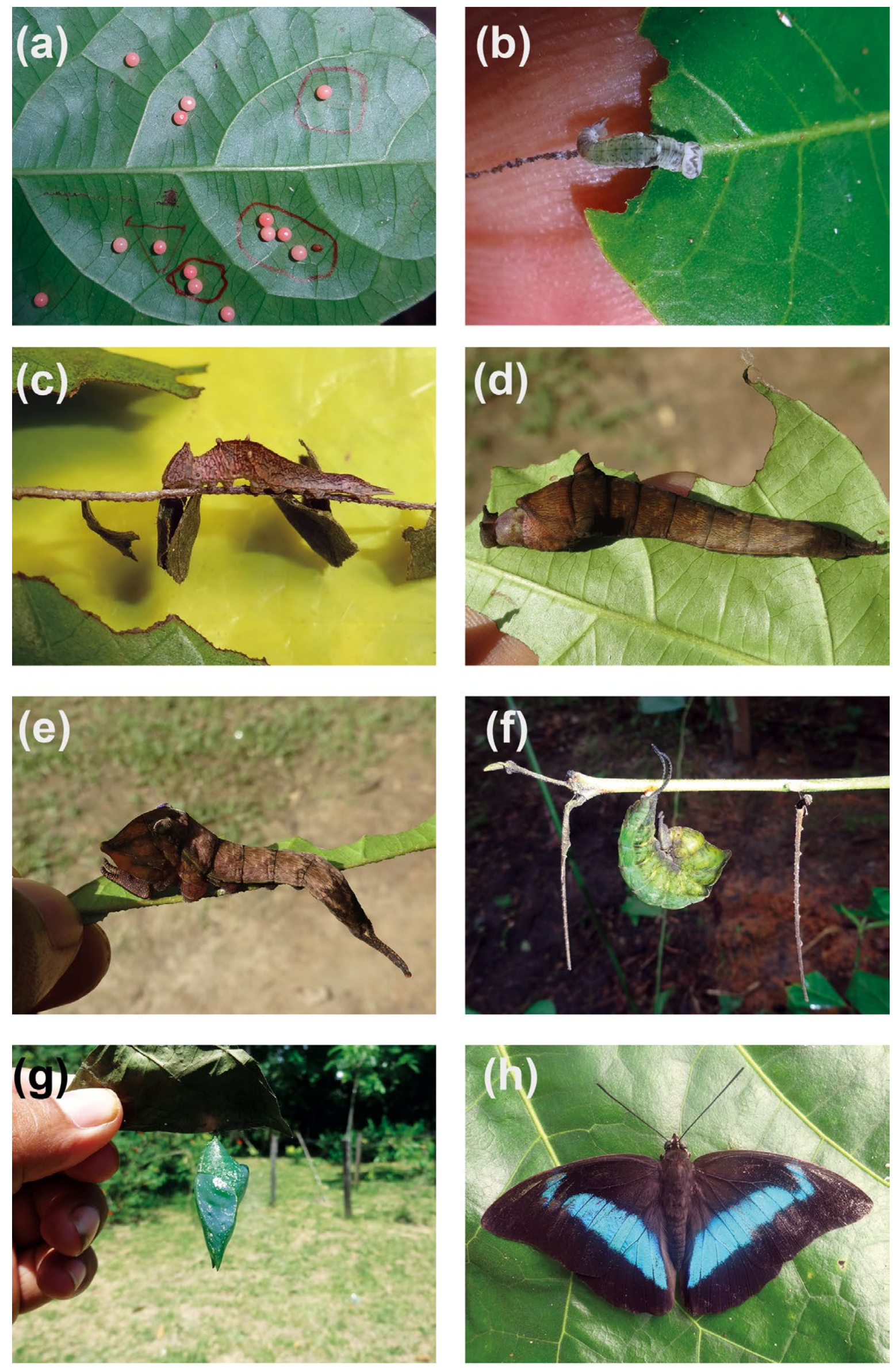

Figura 2. (a) Huevos de Archaeoprepona demophon muson sobre hojas de Siparuna bifida; (b) Larva primer estadío; (c) Larva segundo estadío; (d) Larva cuarto estadío; (e) Larva quinto estadío (f) Prepupa vista lateral adherida a una rama de Siparuna bifida; (g) Pupa vista lateral; (h) Adulto macho vista dorsal. 
partir del extremo caudal y se encorva uniendo la cabeza con el final del cuerpo tornándose totalmente verde olivo y la cola se encorva por encima del sustrato de forma libre. La duración fue de $3.60 \pm 0.51$ días.

- Pupa (Fig. 2g). Es verde olivo moteado de blanco. En el dorso presenta un abultamiento prominente. La duración fue de $14.13 \pm 2.62$ días, menor a lo reportado por González (2006) quien indica $15.40 \pm 1.39$ días con hojas de Rollinia sp. y muy similar a los $14.69 \pm 1.06$ días con hojas de Aniba sp.

El ciclo total desde huevo hasta adulto tiene una duración de $85.40 \pm 4.66$ días y los adultos nacen entre las 9 y $11 \mathrm{~h}$. No existe dimorfismo sexual. Los adultos de los dos sexos son similares, de color negro, en los dos pares de alas presentan una banda azul continua, en el primer par de alas tienen además una mancha curvada azul ubicada en la parte superior muy cerca de la banda azul. La hembra vive $41.00 \pm 6.35$ días y el macho (Fig. 2h) $19.25 \pm 4.49$ días.

Enemigos Naturales.- Se han identificado dos enemigos naturales, una pequeña araña blanquecina que se alimenta de los huevos. Luego que la hembra oviposita, la arańa se aproxima y los consume, dejándolos secos. El segundo enemigo, que se observó en una sola oportunidad, es un hongo que atacó una pupa; luego de sufrir el daño del hongo se tornó de marrón oscuro. El hongo esporula emergiendo de diversas partes de la pupa.

\section{Agradecimientos}

Este trabajo fue financiado por el "Programa Nacional de Innovación para la Competitividad y Productividad, InnóvatePerú" a través del proyecto "Modelos tecnológicos de crianza de 10 especies de mariposas diurnas para su aprovechamiento en bionegocios en la Región Loreto", conducido por el Programa de Investigación en Biodiversidad Amazónica del Instituto de Investigaciones de la Amazonía Peruana. Los autores agradecen a Juan José Bellido por su aporte bibliográfico, revisión y comentarios que enriquecieron los resultados y a George Gallardo Gonzáles por su colaboración con el Sistema de Información Geográfica.

\section{Bibliografía citada}

Amasifuén C. \& R. Zárate 2005. Composición Taxonómica, Ecología y Periodo de Floración de Plantas Leńosas "Dicotiledóneas". Tesis de Biólogo. Universidad Nacional de la Amazonía Peruana. Iquitos, Perú. 397 p.

Beccaloni G., A. Viloria, S. Hall \& G. Robinson. 2008. Catálogo de las plantas huésped de las mariposas neotropicales. Zaragoza: Sociedad Entomológica Aragonesa. 536pp.

Bonfantti D., M.M. Casagrande \& O.H.H. Mielke. 2013. Male genitalia of neotropical Charaxinae: A comparative analysis of character variation. Journal of Insect Science 13(35):1-54. doi: http://dx.doi.org/10.1673/031.013.3501

Calero-Mejía H., I. Armbrecht \& J Montoya-Lerma. 2014. Mariposas diurnas y nocturnas (Lepidoptera: Papilionoidae, Saturniidae, Sphingidae) del Parque Nacional Natural Gorgona, Pacífico Colombiano. Revista de Biología Tropical 62(Suppl.1):317-328. doi: http://dx.doi.org/10.15517/ rbt.v62i0.16351

Campos L. \& J. Ramírez. 2005. Diversidad, patrones de distribución y estructura de comunidades de las mariposas de la Zona Reservada Allpahuayo-Mishana, Loreto, Perú. Tesis de Biólogo. Universidad Nacional de la Amazonia Peruana, Facultad de Ciencias Biológicas. Iquitos, Perú. 89pp.
Checa M.F., A. Barragán, J. Rodríguez \& M. Christman. 2009. Temporal abundance patterns of butterfly communities (Lepidoptera: Nymphalidae) in the Ecuadorian Amazonia and their relationship with climate. Annales de la Société Entomologique de France (N.S.) 45(4):470-486. DOI http://dx.doi.org/10.1080/00379271.2009.10697630

Gentry A. 1993. A Field Guide to the Families and Genera of Woody Plants of Northwest South America (Colombia, Ecuador, Perú) with supplementary notes on herbaceous taxa. Conservation International. Washington, USA. 895pp.

Gonzáles R. 2006. Preferencia de Archeoprepona demophon muson (Fruhstorfer, 1905), Nymphalidae: Charaxinae), por diferentes sustratos de alimentación en condiciones de cautiverio en la microcuenca del río Shilcayo-Tarapoto. Tesis de Ingeniero. Universidad Nacional de San Martín, Tarapoto, Perú. 97pp.

Henao E.R. 2006. Aproximación a la distribución de mariposas del departamento de Antioquia (Papilionidae, Pieridae y Nymphalidae: Lepidóptera) con base en zonas de vida. Boletín Científico. Centro de Museos. Museo de Historia Natural Universidad de Caldas 10: 279-312.

IIAP. 2008. Plan de Negocio de Ecoturismo para la Comunidad Campesina de San Rafael. Proyecto Focal Bosques. Documento Técnico $\mathrm{N}^{\circ}$ 9, Iquitos, Perú. 98pp.

Judd W., C. Campbell, E. Kellogg \& P. Stevens. 1999. Plant Systematics a phylogenetic approach. Sinauer Associates, Inc. Sunderland Massachussets, USA. 464pp.

Lamas G., R.K. Robbins, D.J. Harvey. 1997. Mariposas del alto río Napo, Loreto, Perú (Lepidoptera: Papilionoidea y Hesperioidea). Revista Peruana de Entomología 39:63-74.

Lazzeri M.G., E. Núñez-Bustos \& M.P. Damborsky. 2012. Nuevos registros de Archaeoprepona demophon thalpius (Hübner, [1814]) en las provincias de Corrientes y Chaco, Argentina (Lepidoptera: Nymphalidae, Charaxinae, Preponini). Shilap, Revista de Lepidopterología 40:101-105.

Muyshondt A. 1976. Notes on the life cycle and natural history of butterflies of El Salvador. VII. Archaeoprepona demophon centralis (Nymphalidae). Journal of the Lepidopterists' Society 30(1):23-32.

Ribeiro J., M. Hopkins, A. Vicentini, C. Sothers, M. Costa, J. Brito, M. Souza, L. Martins, L. Lohmann, P. Assuncao, E. Pereira, C. Silva, M. Mesquita \& L. Procopio. 1999. Flora da Reserva Ducke. Guia de identificaçao das plantas vasculares de uma floresta de terrafirme na Amazônia Central. INPA. Manaus, Brasil. 799pp.

Robbins R.K., G. Lamas, O.H.H. Mielke, D.J. Harvey \& M.M Casagrande. 1996. Taxonomic composition and ecological structure of the species-rich butterfly community at Pakitza, Parque Nacional del Manu, Peru, in: Wilson, D. E.; Sandoval, A. (Eds). Manu: The biodiversity of Southeastern Peru. Smithsonian Institution Press, Washington DC. pp. 217-252.

Salazar J. 2011. Notes on the systematic status and distribution of the neotropical butterfly Anaeomorpha splendida Rothschild, 1894 (Lepidoptera: Charaxidae). Boletín Científico. Centro de Museos. Museo de Historia Natural. Universidad de Caldas 15(1):188-205.

Thomas C.D. 1991. Habitat Use and Geographic Ranges of Butterflies from the Wet Lowlands of Costa Rica. Biological Conservation 55: 269-281. DOI https://doi.org/10.1016/0006$3207(91) 90032-5$

Vásquez J., R. Zárate., P. Huiñapi, J. Pinedo, J.J. Ramírez, G. Lamas \& P. Vela. 2017. Plantas alimenticias de 19 especies de mariposas diurnas (Lepidoptera) en Loreto, Perú. Revista Peruana de Biología 24(1):35-42. doi: http://dx.doi.org/10.15381/ rpb.v24i1.13109

Vásquez R. 1997. Flórula de las Reservas Biológicas de Iquitos, Perú. Missouri Botanical Garden Press. St. Louis-USA. 1046 pp.

Vásquez R. \& R. Rojas. 2004. Plantas de la Amazonía Peruana Clave para Identificar las Familias de Gymnospermae y Angiospermae. Arnaldoa Edición Especial: 1-261. 